\title{
Effect of parameter selection on different topological structures for Particle Swarm Optimization algorithm
}

\author{
Daniele Peri ${ }^{{ }^{*}}$ \\ ${ }^{1}$ CNR-IAC - National Research Council - Istituto per le Applicazioni del Calcolo "Mauro Picone" - Via dei \\ Taurini 19, 00185 Rome, Italy \\ *Email address for correspondence: d.peri@iac.cnr.it \\ Communicated by Giorgio Fotia \\ Received on 03 05, 2019. Accepted on 10 21, 2019.
}

\begin{abstract}
Particle Swarm Optimization is an evolutionary optimization algorithm, largely studied during the years: analysis of convergence, determination of the optimal coefficients, hybridization of the original algorithm and also the determination of the best relationship structure between the swarm elements (topology) have been investigated largely. Unfortunately, all these studies have been produced separately, and the same coefficients, derived for the original topology of the algorithm, have been always applied. The intent of this paper is to identify the best set of coefficients for different topological structures. A large suite of objective functions are considered and the best compromise coefficients are identified for each topology. Results are finally compared on the base of a practical ship design application.
\end{abstract}

Keywords: Optimization, Heuristic methods, Evolutionary computation, Particle Swarm Optimization

AMS subject classification: $90 \mathrm{C} 30,65 \mathrm{~K} 10,90 \mathrm{C} 26,90 \mathrm{C} 56$

\section{Introduction}

Since its introduction in 1995 [1], Particle Swarm Optimization (PSO) algorithm received a lot of attention, and a large number of studies rapidly came out, as reported in [2]. The main investigated elements were the coefficients ruling the algorithm, the number of elements forming the swarm, their initial position and speed, the hybridization with other optimization algorithms and also the scheme for the relationship between the elements of the swarm [3] [4] (topology). However, the same coefficients identified as preferable for the original configuration of the algorithm have been always adopted, also when a strong modification were proposed: as a consequence, a systematic study on the relationship between topologies and coefficients is substantially missing. The main purpose of this article is to focus on this aspect, analyzing the performances of the algorithm by varying together the topology and the coefficients of the algorithm.

To do that, a first overview of the logic of the algorithm is presented, identifying some possible topologies to be applied to the original PSO algorithm. Then, a wide investigation on the best coefficient for each different topology is performed: algebraic functions are adopted in this phase as objective functions. The best combinations are finally compared by solving a realistic industrial optimization problem. Concluding remarks and possible future research directions are proposed at the end.

\section{Swarm logic}

Since PSO is inspired by the behavior of a swarm of bees, it could be interesting to compare it with a typical mathematical model describing the dynamics involving the individuals of a swarm [5], that is

$$
m \frac{d v_{i}}{d t}=\left(\alpha-\beta\left\|v_{i}\right\|^{2}\right) v_{i}-\sum_{i \neq j} \nabla U\left(\left\|x-x_{i}\right\|\right)
$$


where $m$ is the mass, $x_{i}$ and $v_{i}$ are the position and the speed of the $i^{\text {th }}$ element of the swarm, $\alpha$ and $\beta$ are regulating the relationship between between force and speed and $U$ is a potential function, classically the generalized Morse potential

$$
U\left(x_{i}\right)=\sum_{i \neq j}\left[C_{r} e^{-\left|x_{i}-x_{j}\right| / \ell_{r}}-C_{a} e^{-\left|x_{i}-x_{j}\right| / \ell_{a}}\right]
$$

with $\ell_{a}$ and $\ell_{r}$ representing the attractive and repulsive potential ranges, and $C_{a}$ and $C_{r}$ their amplitudes. This mathematical model, from now on Fully Informed (FI), describes the relationship between the elements of the swarm: here a combination of repulsive and attractive forces is creating the swarm and the relative weight between the two is determining its behavior. If compared to the FI model, the classical PSO structure shows only few analogies. In [1], the swarm iteration is defined as

$$
\begin{gathered}
v_{i}^{k}=w v_{i}^{k-1}+c_{1} r_{1}\left(x_{i}^{b}-x_{i}^{k-1}\right)+c_{2} r_{2}\left(x_{o}^{b}-x_{i}^{k-1}\right) \\
x_{i}^{k}=x_{i}^{k-1}+v_{i}^{k}
\end{gathered}
$$

where $x_{i}^{k}$ and $v_{i}^{k}$ are the position and the speed of the $i^{\text {th }}$ element of the swarm at the $k^{\text {th }}$ iteration, $x_{i}^{b}$ is the best position ever visited by $x_{i}$, and $x_{o}^{b}$ is the overall best among all the $x_{i}^{b}$ 's. $r_{1}$ and $r_{2}$ are random coefficients and $w$ is the inertia term. An initial population of $N$ elements $\left\{x_{1}^{1},[\ldots], x_{N}^{1}\right\}$ is defined (randomly or by using a spatially uniform distribution [6]), the objective function is computed for every $x_{i}^{1}$ and the best value for the $i^{t h}$ element is stored in $x_{i}^{b}$. Also $x_{o}^{b}$ is fixed. The new speed of each element is computed using equation 2, the swarm is moved accordingly and a new position for each element of the swarm $x_{i}^{2}$ is obtained, and so on. In this study, a deterministic version of PSO (DPSO), is applied. In DPSO, the random coefficients $r_{1}$ and $r_{2}$ are deleted, and equation 2 now reads:

$$
v_{i}^{k}=w v_{i}^{k-1}+c_{1}\left(x_{i-1}^{b}-x_{i}^{k-1}\right)+c_{2}\left(x_{o}^{b}-x_{i}^{k-1}\right)
$$

Elsewhere, PSO optimization would be repeated a large number of times in order to produce a statistics of the global minimum. By the way, DPSO is found to be preferable to PSO in practical applications [7].

If we compare equation 4 with equation 1, we can observe the following differences for PSO:

1. the repulsive potential of equation 1 is not included;

2. every element $x_{i}^{k}$ is communicating with the overall best element $x_{o}^{b}$ but not viceversa.

3. the attractive potential of equation 4 is linear, but for each element it is applied considering a couple of points only $\left(x_{i}^{b}\right.$ and $\left.x_{o}^{b}\right)$;

4. $x_{i}^{b}$ and $x_{o}^{b}$ are positions visited by the swarm, not necessarily included into the current swarm. As a consequence, 4 describes the motion of $N$ isolated individuals interacting with two attraction points whose position is changing in time.

While for the FI model the information flow involves every element in both the directions, in PSO only individual interactions in a single direction are included. The topological structure is a star type (see figure 1): the second term in equation 4 refers to the element itself, so that a connecting line on the graph is missing, and the third term points to the overall best (the central point of the star). The central element has no interactions with the others, and its new velocity is updated by using information coming from the element itself.

In order to check if a different communication scheme led to better performances, various alternatives have been proposed in literature. In [3], four different topologies are considered: token ring, wheel, star and random (see figure 1). Some hybrid version of the topologies have been also investigated, mixing together wheel and ring, defining a huge number of intermediate subversions. Unfortunately, only four test functions have been adopted: the results cannot be extrapolated to a general case, but the sensitivity of the algorithm to the topological scheme is evidenced. 
The FI model (Fully Informed PSO - FIPS) has been also considered in [8]), obtaining mixed results: there is not a clear superiority of FIPS over PSO. Several other studies demonstrate that FIPS is not the best configuration [9], and for this reason it will not be further considered.

In [10], starting from the FI topology and using an optimization procedure, a subset of topologies has been identified. Unfortunately, optimal topology is targeted for a prescribed number of swarm element, and this is a strong limitation for a general-purpose application. General conclusions are that the increase of the connectivity is improving the convergent attitude of the algorithm, but this is not a guarantee of the determination of the global minimum.

Another promising topology is the circular structure (token ring), proposed in [10] and further analyzed, among the others, in [11], showing some improvements with respect to classical PSO.

Summarizing, among the different topologies analyzed in the current literature, only the ring topology shows some improvements with respect to the original star scheme, and for this reason they are both considered for the optimization of the coefficients. For the ring topology, four variants are here proposed:

1. The $i^{t h}$ element moves toward the $\left(i+1^{t h}\right)$ personal best element, ordered on the base of their objective function value. The overall best is attracted by the worst element in the ring $\left(\operatorname{ring}_{f}^{a}\right)$.

2. As $\operatorname{ring}_{f}^{a}$, but the overall best is repelled by the worst element $\left(\operatorname{ring}_{f}^{r}\right)$.

3. The $i^{\text {th }}$ element moves toward the closer element in the swarm: distance is measured using the current position, and the overall best is attracted by the last element in the $\operatorname{ring}\left(\operatorname{ring}_{x}^{a}\right)$.

4. As $\operatorname{ring}_{x}^{a}$, but the overall best is repelled by the last element $\left(\operatorname{ring}_{x}^{r}\right)$.

In the variant $\operatorname{ring}_{f}^{a}$ and $\operatorname{ring}_{f}^{r}$, the elements are labeled in descending order of the objective function value. In the variant $\operatorname{ring}_{x}^{a}$ and $\operatorname{ring}_{x}^{r}$, number $\mathrm{N}$ is assigned to the best element: since then, proceeding in reverse order, number $i-1$ is given to the element closer to $i$ (basing on the Euclidean distance). Once a label is assigned, the element is excluded from the successive search. Since we are considering a minimization problem, $x_{N}$ is always the overall best element. The order of the elements is dynamically changing as a consequence of the movement of the swarm. In the second block of equation 4, the sign may change for the best element, depending if we are enforcing attraction or repulsion.

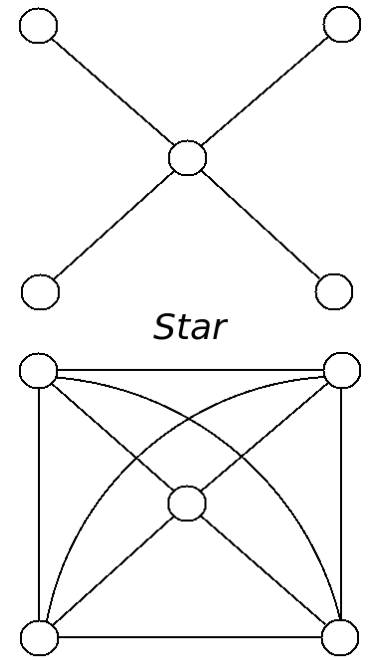

Fully Informed

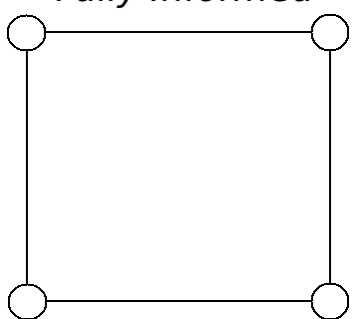

Token ring

Figure 1: Representation of three common topological schemes: Star, Full, and Token Ring.

The circle represents the element of the swarm, a connection implies a flow of information between the two. The information flow can be one- or two-ways.

\section{Numerical experiments}

In order to find the optimal coefficients $\left\{c_{1}, c_{2}, w\right\}$ for the different topologies, 53 analytical functions are used in this test: they are listed in Table 5. For every triplet of the coefficients we can apply DPSO on every analytical function $f^{i}$, finding the minimum value $f_{\text {best }}^{i}\left(c_{1}, c_{2}, w\right)$. The optimization of the coefficients of DPSO is performed minimizing the objective function $F O$ defined as

$$
F O\left(c_{1}, c_{2}, w\right)=\frac{\sum_{i=1}^{53} f_{b e s t}^{i}\left(c_{1}, c_{2}, w\right)}{53}
$$

Since $f_{\text {best }}^{i}$ is always the same for every function (zero), there is no need of a normalization. Minimization of $F O$ is performed by using a Direct Search Algorithm (DS) [12]. 

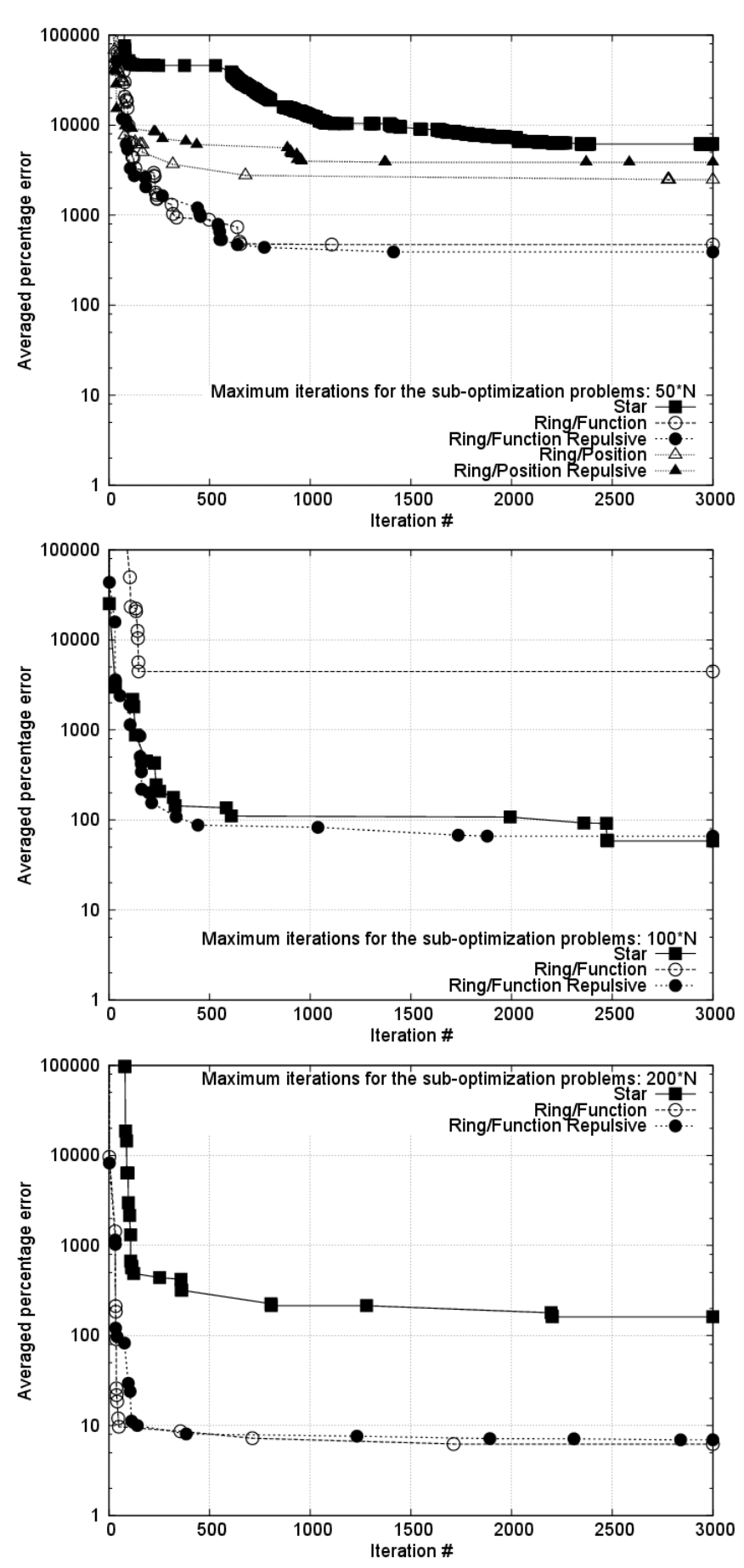

Figure 2: Convergence history of the global error associated with a specific triplet of DPSO coefficients. Each graph is obtained by varying the maximum number of iterations permitted during the suboptimization problem: from left to right, the DPSO is stopped after $50 \times N D V, 100 \times N D V$ and $200 \times N D V$ iterations, where $N D V$ is the number of design variables for the considered analytical function.
Both DPSO and DS are stopped when a prescribed number of iterations has been performed. The maximum number is $N_{M A X} x N D V$ : it has been selected, in order to give more opportunities to the objective functions with a larger number of design variables $N D V$. A preliminary test has been performed by stopping the DS setting $N_{M A X}^{D S}$ to 50. The purpose of this test is to reduce the number of candidate topologies: in the successive tests, the convergence of the DS will be based on the range of the search, and not on the number of iterations, in order to reach a complete convergence. Results are reported at the left end of the figure 2, using the global error (measured as the average of the percentage difference between the achieved minimum and the true global minimum for all the analytical function): the classical topology is largely improved by the ring topology, with a clear preference for the versions based on the objective function value rather than the position. For this reason, in the following examples the two topologies based on the distance $\left(\operatorname{ring}_{x}^{a}\right.$ and $\operatorname{ring}_{x}^{r}$ ) will be no more considered.

Both the new tests with $N_{M A X}$ equal to 100 and 200 are substantially confirming the previous results. The only difference is related to the absolute value of the average error, decreasing of about one order of magnitude when $N_{M A X}$ is doubled. Increasing $N_{M A X}$ to 400 does not provide further improvement. Results are reported in Table 3. In the following, the option with $N_{M A X}=200$ will be adopted.

Looking at the coefficients obtained with $N_{M A X}=200$, they are smaller than the classical PSO coefficients. The difference between $c_{i}$ and $w$ is larger for the star topology, while the same behavior is present, but less pronounced, in the ring topologies. No substantial differences are observed between the optimal coefficients of $\operatorname{ring}_{f}^{a}$ and $\operatorname{ring}_{f}^{r}$.

An example for a specific function is reported in figure 3. Here the full convergence history for the "Six Humps Camel Back" function (the first in Table 5) is shown: the objective function value is plotted as a function of the iteration number. We can observe that all the different versions of the algorithm are able to minimize the function. The faster topology is $\operatorname{ring}_{f}^{a}$, while the star topology is the slower one.

\section{Ship design application}




\begin{tabular}{|l|l|l|l|}
\hline Topology & $\mathrm{c}_{1}$ & \multicolumn{1}{|c|}{$\mathrm{c}_{2}$} & $w$ \\
\hline & \multicolumn{3}{|c|}{$200 \times N D V$} \\
\hline Star & 0.183 & 0.242 & 0.807 \\
\hline $\operatorname{Ring}_{f}^{a}$ & 0.456 & 0.369 & 0.673 \\
\hline $\operatorname{Ring}_{f}^{r}$ & 0.441 & 0.307 & 0.693 \\
\hline & \multicolumn{3}{|c|}{$100 \times N D V$} \\
\hline Star & 0.409 & 0.287 & 0.662 \\
\hline $\operatorname{Ring}_{f}^{a}$ & 0.411 & 0.054 & 0.570 \\
\hline $\operatorname{Ring}_{f}^{r}$ & 0.353 & 0.055 & 0.596 \\
\hline & \multicolumn{3}{|c}{$50 \times N D V$} \\
\hline Star $^{r}$ & 0.082 & 0.079 & 0.184 \\
\hline $\operatorname{Ring}_{f}^{a}$ & 0.481 & 0.053 & 0.408 \\
\hline $\operatorname{Ring}_{f}^{r}$ & 0.421 & 0.051 & 0.415 \\
\hline
\end{tabular}

Table 1: Numerical value of the DPSO optimal coefficients for the investigated topologies and for different stopping criteria.

For the verification of the obtained coefficients (reported in Table 3) on a realistic optimization problem, a ship design application has been considered. The geometry of a sailing yacht is here optimized by using DPSO with all the previous best topologies in combination with their optimal coefficients. Objective function if represented by the total resistance of the ship in straight course at the speed of 10 knots in calm water, and it is evaluated by means of a RANS solver [13]. Parameterization of the hull surface is performed by applying the Free Form Deformation (FFD) approach [14], here using five parameters. One parameter is shifting the midsection fore and aft, two parameters are changing the shape of the transverse section of the hull, the last two are acting vertically, one at the bow and one at the stern. Maximum movement of the control points of the FFD is limited to 0.5 meters. The only constraint enforces the total weight of the ship, limited in between 35 and 36 tonnes, being 35 tonnes the original weight of the ship.

The three best topologies have been used, and results are reported in Figure 4. In this picture, the convergence of the optimization problem is observed by plotting the percentage reduction of the total resistance as a function of the iteration number. Topology indicated as $\operatorname{ring}_{f}^{a}$ gives the best results. All the other topologies are separated by a percentage difference in between 2 and $5 \%$.

Improvement on the objective function is of the order of $40 \%$, reducing total resistance from 921 to $478 \mathrm{~N}$. This improvement is surely exaggerated, and substantially due to the coarse grid density adopted 
in order to reduce the computational time: anyway, the coarseness of the computational grid is not influencing the validity of the optimization tests. The reduction of the predicted total resistance is also reflected on the global characteristics of the predicted wave field, reported in Figure 5. A small increase of the wave elevation at the bow, together with the depression at the first thought, is observed for the optimal hull: this could be intuitively regarded as a negative point. But if we observe the wave elevation astern, we can see a really strong reduction obtained with the optimal hull, and this is probably the reason of the reduced value of the total resistance.

\section{Conclusions}

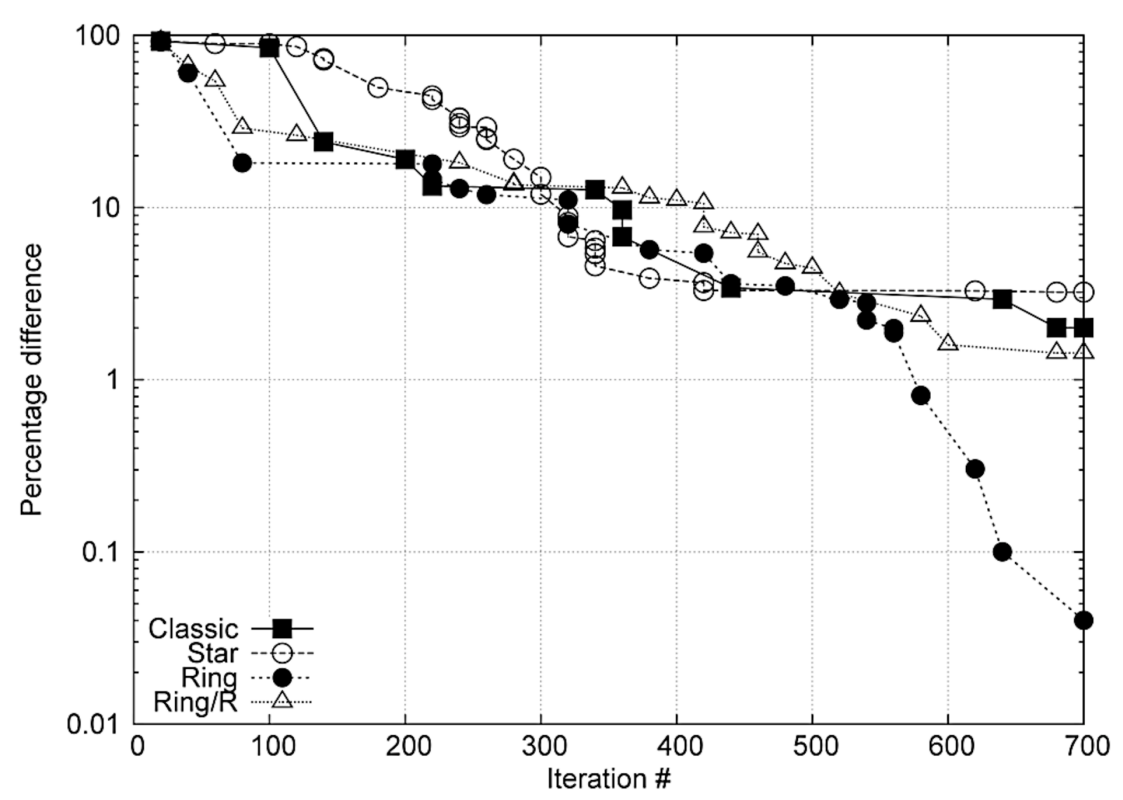

Figure 4: Percentage difference between the current value of the objective function and a reference value close to the overall best optimal as a function of the iteration number for the standard DPSO algorithm with different topologies and coefficients. Percentage difference is reported in logarithmic scale.
Five different topologies, listed at the end of section 2, have been investigated for the DPSO algorithm. Topology has been confirmed to be a crucial element for the improvement of the overall characteristics of the algorithm, as already observed in literature, and significant results have been produced in this sense, detecting optimal DPSO coefficients and preferable topologies. A further investigation should be performed on more advanced implementation of the DPSO algorithm (i.e. iPSO, [6]), where local and global search algorithms are coupled together: a simple comparison could be performed by using the here described topologies and coefficients, but also a variation of the topology of iPSO in combination with a variation of the main parameters might represents an interesting test

\section{Acknowledgements}

The author is grateful to Dr. Andrea Di Mascio, CNR-IAC, for the use of the RANS solver MGShip. 


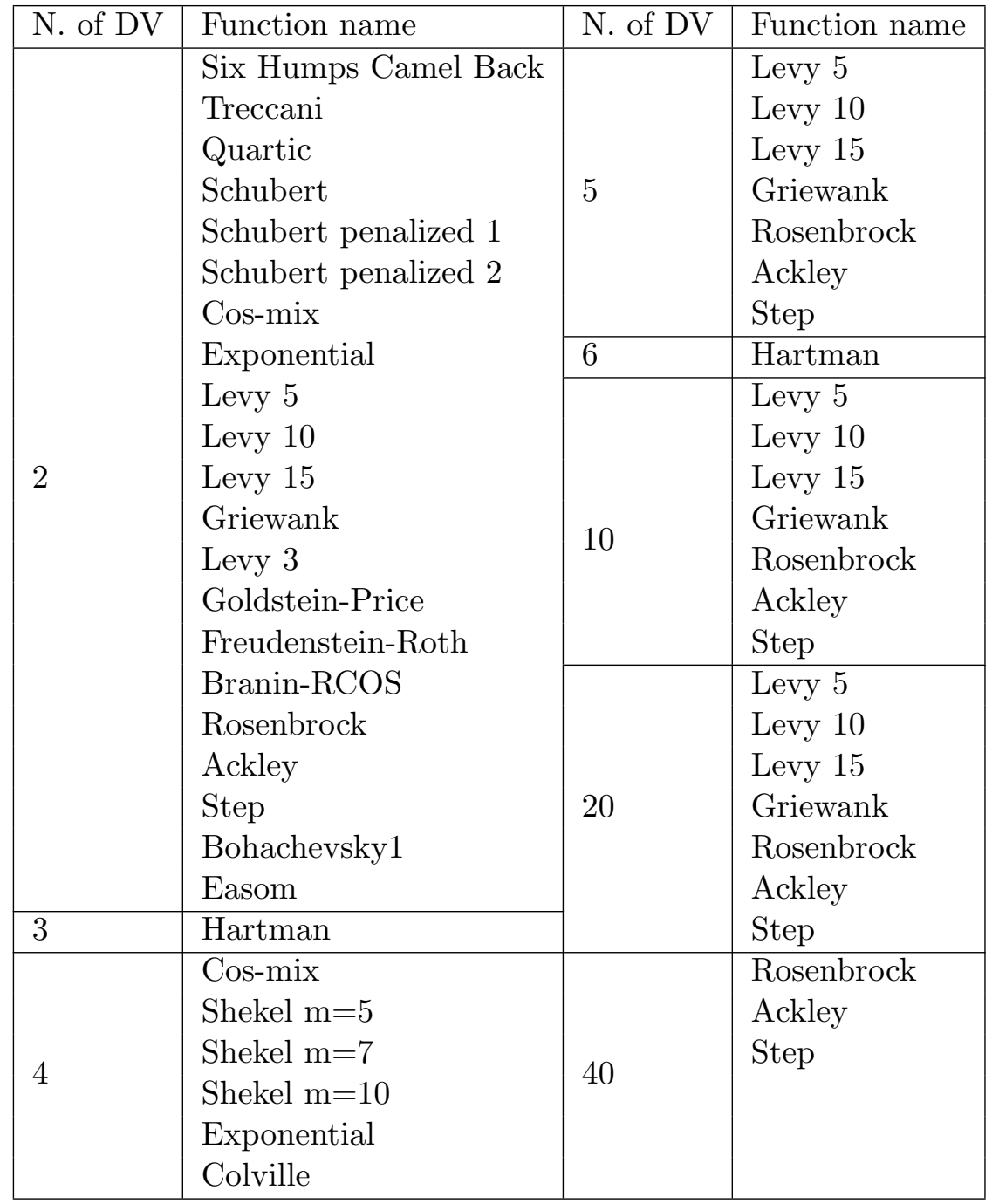

Table 2: List of the analytical test functions adopted for the optimization of the PSO coefficients. The definitions of the functions together with the limits of the design space can be find at http://wwwoptima.amp.i.kyoto-u.ac.jp/ 


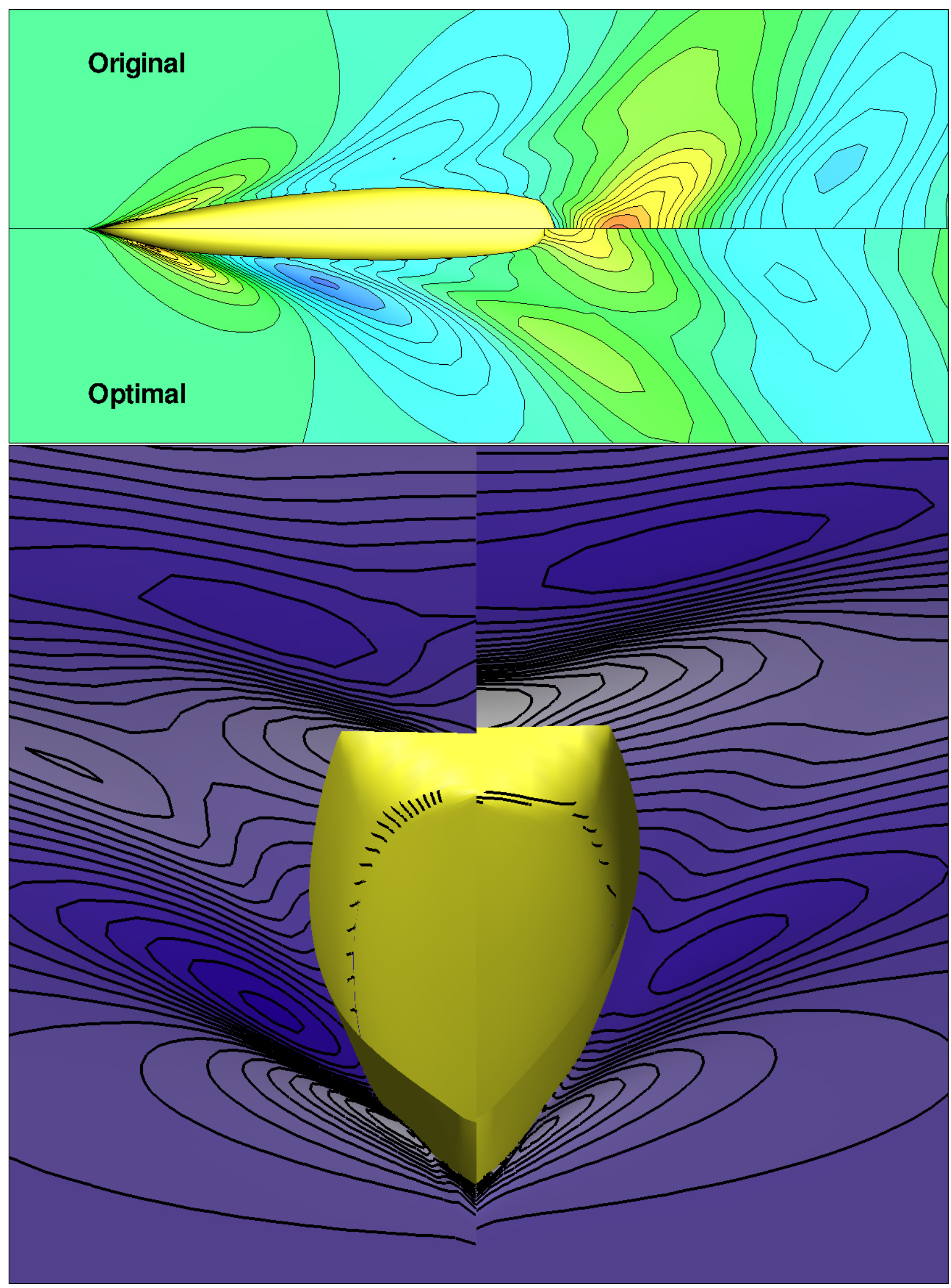

Figure 5: Perspective and plane view of the original and optimized hull shape: in the top figure, on right the original hull shape, on left the optimal one. An increase of the bow wave is observed for the optimal hull together with a strong reduction of the stern wave system: the overall effect is a reduction of objective function of about $40 \%$. 


\section{References}

1. J. Kennedy and R. Eberhart, Particle swarm optimization, in Proc. IEEE Int'l. Conf. on Neural Networks, 1995.

2. Y. Zhang, S. Wang, and J. Gelin, A comprehensive survey on particle swarm optimization algorithm and its applications, Mathematical Problems in Engineering, vol. 2015, no. ID 931256, pp. 1-38, 2015.

3. J. Kennedy, Small worlds and mega-minds: Effects of neighborhood topology on particle swarm optimization, in Proc. IEEE Int'l. Conf. on Evolutionary Computations - CEC '99, vol. 3, pp. 1931-1938, World Scientific, 1999.

4. P. Suganthan, Particle swarm optimizer with neighborhood operator, in Proc. IEEE Int'l. Conf. on Evolutionary Computations - CEC '99, vol. 3, pp. 1958-1962, 1999.

5. M. D'Orsogna, Y. C. Y., A. Bertozzi, and L. Chayes, Self-propelled particles with soft-core interactions: patterns, stability and collapse, Phys. Rev. Lett., vol. 96, no. 10, 2006.

6. D. Peri, An inner-point modification of pso for constrained optimization, Engineering Computations, vol. 32, no. 7, pp. 2005-2019, 2015.

7. D. Peri, M. D. M., and G. Fasano, Comparison between deterministic and stochastic formulations of particle swarm optimization, for multidisciplinary design optimization, in 14th AIAA/ISSMO Multidisciplinary Analysis and Optimization Conference, vol. AIAA Paper 2012-5523, pp. 71-78, AIAA, 2012.

8. R. Mendes, J. Kennedy, and J. Neves, The fully informed particle swarm: simpler, maybe better, IEEE Transactions on Evolutionary Computation, vol. 8, no. 3, pp. 204-210, 2004.

9. M. M. de Oca and T. S. T., Convergence behavior of the fully informed particle swarm optimization algorithm, in GECCO '08 Proceedings of the 10th annual conference on Genetic and evolutionary computation, pp. 71-78, 2008.

10. R. Kennedy and R. Mendes, Population structure and particle swarm performance, in Proc. IEEE Int'l. Conf. on Evolutionary Computations - CEC '02, vol. 2, pp. 1671-1676, 2002.

11. X. Li, Adaptively choosing neighborhood bests using species in a particle swarm optimizer for multimodal function optimization, in Genetic and Evolutionary Computation - GECCO 2004, pp. 105-116, 2004.

12. C. Perttunen, Geometric approach to feasible region division in constrained global optimization, in IEEE International Conference on Systems, Man, and Cybernetics 'Decision Aiding for Complex Systems', vol. 1, pp. 582-590, 1991.

13. A. D. Mascio, R. Broglia, and B. Favini, A Second Order Godunov-Type Scheme for Naval Hydrodynamics, pp. 253-261. Boston, MA: Springer US, 2001.

14. T. Sederberg and S. Parry, Free-form deformation of solid geometric models, in Proceedings of the 13th Annual Conference on Computer Graphics and Interactive Techniques, SIGGRAPH 1986, vol. 2(4), pp. 151-160, 1986. 